\title{
PREVALENCIA DE CHLAMYDIA TRACHOMATIS EN EMBARAZADAS DURANTE EL PRIMER TRIMESTRE EN UNA INSTITUCIÓN PRIVADA DE LA CIUDAD DE CÓRDOBA.
}

\author{
PREVALENCE OF CHLAMYDIA TRACHOMATIS IN PREGNANT WOMEN DURING THE FIRST \\ TRIMESTER IN A PRIVATE INSTITUTION IN CÓRDOBA.
}

\section{Agostina Zucotti ${ }^{1}$, Lucrecia Bolaño², Fabiana Alicia Berruezo ${ }^{3}$, Susana Vitozzi ${ }^{4}$, Marina Bottiglieri ${ }^{5}$.}

\section{Resumen:}

Introducción: En las embarazadas, la presencia de $C$. trachomatis puede provocar ruptura prematura de membrana, endometritis puerperal y parto prematuro. El curso asintomático de la infección hace más difícil su diagnóstico clínico. Objetivos: Conocer la prevalencia de Chlamydia trachomatis en las embarazadas que cursan su primer trimestre y determinar si existen factores asociados a la presencia de dicha bacteria. Material y métodos: Estudio prospectivo, descriptivo, transversal y correlacional, realizado en la Clínica Universitaria Reina Fabiola, Córdoba, Argentina desde octubre 2016 hasta abril 2017. La detección de C. trachomatis se realizó por técnica de Real Time PCR en una muestra de primer chorro de orina. Resultados: se estudiaron 350 embarazadas. La prevalencia global fue del $2 \%$, con un $13,3 \%$ en las menores de 25 años ( $p<0,001)$. La misma observación se notó con respecto al nivel educativo, con una prevalencia superior en las que tenían sólo educación primaria, $18,2 \%$ ( $p<0.001)$, en las mujeres que presentaron algún signo 0 síntoma ginecológico bajo, 6,8\% $(p<0,001)$ y en aquellas pacientes cuyo inicio de la actividad sexual fue antes de los 18 años, $3,3 \%(p<0,030)$. Conclusión: A la luz de estos hallazgos se sugiere realizar la búsqueda de $C$. trachomatis como parte del screening prenatal en todas las mujeres embarazadas menores de 25 años. El sistema de salud argentino debería tomar en cuenta esta problemática para lograr un control racional de las infecciones causadas por esta bacteria.

Palabras clave: Chlamydia trachomatis; embarazo; prevalencia; RT PCR; primer chorro de orina.

\section{Abstract:}

Introduction: The presence of $C$. trachomatis in pregnant women can cause premature rupture of membranes, postpartum endometritis and preterm birth. Clinical diagnosis is more difficult among those who have an asymptomatic infection. Objective: know the prevalence of Chlamydia trachomatis in pregnant women in their first trimester and to detect if there are any factors associated with this bacterium. Material and methods: Prospective, descriptive, cross sectional and correlational study performed at Clínica Universitaria Reina Fabiola, in Córdoba, between October 2016 and April 2017. C. trachomatis was detected through the Real Time PCR technique in first void urine samples. Results: 350 pregnant women were studied. The global prevalence was of $2 \%$ with a $13,3 \%$ in women under 25 years old $(p<0,001)$. A similar pattern was observed with respect to the educational level, with a higher prevalence on those who only had primary education, $18,2 \%(p<0.001)$; in women who presented a low gynecological sign or symptom $6,8 \%(p<0,001)$ and in those patients whose sexual initiation was before 18 years old, $3,3 \%(p<0,030)$. Conclusion: It is advisable to implement $C$. trachomatis screening as part of the pre natal screening in all pregnant women under 25 years old. Argentinian health care system should take this problem into account in order to achieve a rational control over the infections caused by this bacterium.

Keywords: Chlamydia trachomatis; pregnancy, prevalence; RT PCR; first void urine.

1 Bioquímica. Servicio de Microbiología. Clínica Universitaria Reina Fabiola Córdoba. Argentina. Email de contacto: aguitozucotti3@gmail.com.

2 Médica. Servicio de Ginecología y Obstetricia Clínica Universitaria Reina Fabiola Córdoba. Argentina. Email de contacto: lucre0507@hotmail.com.

3 Especialista en Bacteriología. Servicio de Microbiología. Clínica Universitaria Reina Fabiola Córdoba. Argentina. Email de contacto: fabianaberruezo@hotmail.com.

4 Especialista en Inmunología. Laboratorio LACE- Servicio de Biología Molecular. Email de contacto: -susana.vitozzi@gmail.com.

5 Doctora en Medicina. Especialista en Microbiología. Jefa del Servicio de Microbiología Clínica Universitaria Reina Fabiola Córdoba. Argentina. Email de contacto: marinabottiglieri@gmail.com 


\section{Introducción}

La infección genital por Chlamydia trachomatis es considerada en la actualidad una de las causas más frecuentes de infección de transmisión sexual (ITS) a nivel mundial, estimándose 131 millones de nuevos casos cada año ${ }^{(1)}$.

En las mujeres causa cervicitis, uretritis, salpingitis y enfermedades relacionadas con la reproducción; su presencia puede causar enfermedad inflamatoria pélvica y otro tipo de infecciones genitales altas que dejan como secuela cicatrices o adherencias en las trompas de Falopio. En las embarazadas, puede provocar abortos espontáneos, embarazos ectópicos, ruptura prematura de membrana, endometritis puerperal y parto prematuro ${ }^{(2,3)}$.

Se estima que hasta un $70-75 \%$ de los casos son asintomáticos ${ }^{(4,5)}$.El riesgo de adquirir esta infección es dos veces mayor en mujeres menores de 25 años ${ }^{(6)}$. Un posible mecanismo por el cual se produce ruptura prematura de membrana es la producción de prostaglandinas, que estimulan la contractibilidad uterina, así también colagenasas y proteasas que llevan al debilitamiento de las membranas ${ }^{(7)}$. Aproximadamente dos tercios de los neonatos nacidos por parto vaginal de madres portadoras se pueden infectar al nacer. Un $30-$ $50 \%$ de los recién nacidos de madres no tratadas desarrollan conjuntivitis y un $10-20 \%$, neumonías ${ }^{(8)}$. Menos frecuentemente se presentan casos de otitis media y faringitis. Además, la infección neonatal por $C$. trachomatis puede provocar secuelas a largo plazo como la enfermedad pulmonar obstructiva crónica (9). Los datos epidemiológicos sugieren que las embarazadas son un grupo importante a tener en cuenta para vigilar la presencia de esta bacteria. El examen prenatal, según lo recomendado por el CDC (Centers for Disease Control and Prevention), es beneficioso no sólo para disminuir la morbilidad en las mujeres, sino también para prevenir la infección vertical y la transmisión horizontal ${ }^{(10,11)}$. En Estados Unidos la infección por $C$. trachomatis durante el embarazo ocupa el tercer lugar, después de la vaginosis bacteriana y el virus Herpes simplex $2^{\left({ }^{(10)}\right.}$. La tendencia mundial de las tasas de prevalencia de C.trachomatis va en aumento cada año, con excepción de los países donde se implementaron programas de detección temprana y tratamiento oportuno así como la educación para la prevención de las ITS. Finlandia, Dinamarca, EEUU y Australia lograron una reducción importante de la infección y de sus complicaciones ${ }^{(12,13)}$.

En Argentina los datos disponibles son escasos. Los valores de prevalencia comunicados en la población adolescente general fue del 8,7\% en Córdoba Capital ${ }^{(14)}$ y del 3,5\% en los ingresantes universitarios en la ciudad de Bahía Blanca, Buenos Aires ${ }^{(15)}$.

En América Latina, se han reportado prevalencias en embarazadas del 5,9\% en Chile ${ }^{(16)}, 10 \%$ en Lima, Perú ${ }^{(17)}$ y $12,3 \%$ en el sur de Brasil ${ }^{(18)}$. En Buenos Aires, se reportó en el año 2001 una prevalencia del $2,5 \%$ en mujeres asistidas en un hospital de esa provincia; este grupo de estudio incluyó sólo pacientes sintomáticas ${ }^{(19)}$. El curso frecuentemente asintomático de la infección hace más difícil su diagnóstico clínico, con lo cual se retrasa o no se instaura el tratamiento.

\section{Objetivos}

Conocer la prevalencia de Chlamydia trachomatis en embarazadas que cursan el primer trimestre y determinar si existen factores asociados a la presencia de dicha bacteria.

\section{Pacientes y Métodos}

Se realizó un estudio prospectivo, descriptivo, transversal y correlacional, en el período comprendido desde octubre del 2016 hasta abril del 2017 en la Clínica Universitaria Reina Fabiola, Córdoba, Argentina. Se estudiaron 350 embarazadas que estaban cursando el primer trimestre. Los criterios de exclusión fueron: mujeres que no estaban dentro de su primer trimestre de embarazo o que hubieran recibido tratamiento antibiótico en los últimos 7 días previos a la recolección de la muestra.

Se confeccionó una ficha epidemiológica que las pacientes completaron al momento de la realización del estudio. Los factores evaluados fueron edad, nivel de instrucción, edad de inicio de las relaciones sexuales, número de parejas sexuales en el último año, antecedentes de ITS, utilización de preservativos en relaciones sexuales anteriores al embarazo, síntomas y signos de infección genital baja y antecedentes gineco-obstétricos (embarazos previos, número de hijos, abortos previos, embarazos ectópicos).

A cada paciente se le solicitó una muestra del primer chorro de orina con una retención mínima de 3 horas, obtenida en un frasco estéril y conservada a $4^{\circ} \mathrm{C}$ hasta su procesamiento, no mayor a las 48 horas. 
Se realizó la detección de la bacteria utilizando la técnica de Real Time PCR (RT PCR) (Primerdesign ${ }^{\mathrm{TM}}$ genesig( $\left.{ }^{\circledR}\right)$. Se amplifica la secuencia específica del gen helicasa del plásmido críptico (DnaB) con un control interno para la detección de beta actina para confirmar la presencia de celularidad en la muestra.

Para llevar a cabo el análisis estadístico de los datos, se realizaron tablas de frecuencia con el fin de observar la distribución de las diferentes variables utilizando la pruebas chi cuadrado de independencia y prueba t de diferencia de medias; para observar cuáles fueron los factores que mejor predecían los resultados, se realizaron regresiones logísticas múltiples.

Este trabajo fue aprobado por el Comité de Ética de la institución. Las pacientes firmaron un consentimiento y a aquellas con un resultado positivo para la detección de Chlamydia trachomatis, se les administró tratamiento antibiótico a ellas y a sus parejas en el marco del manejo ético de la situación.

\section{Resultados}

Fueron estudiadas 350 embarazadas (edad media 31,5 años, rango 16-47). Dos de ellas se excluyeron del estudio por presentar negativo el control interno en la muestra de primer chorro de orina. $C$. trachomatis fue detectada en $7 / 348$ mujeres, con una prevalencia global del $2 \%$. La mediana de edad de las mujeres $C$. trachomatis positivas fue de 21 con un rango de 17 a 31 .

La prevalencia de la infección fue mayor en mujeres más jóvenes, siendo del 13,3\% en las menores de 25 años y del $0,9 \%$ en las mayores de esa edad $(p<0,001)$. La misma observación se notó con respecto al nivel educativo, con una prevalencia superior en las que tenían sólo educación primaria, 18,2\% vs 7,7\% en pacientes con niveles educativos superiores $(p<0.001)$; en las mujeres que presentaron algún signo 0 síntoma ginecológico bajo, que incluyeron dispareunia, leucorrea, prurito o ardor al orinar, $6,8 \%(p<0,001)$ y en aquellas pacientes cuyo inicio de la actividad sexual fue antes de los 18 años, $3,3 \%(p=0,03)$.

De las 348 pacientes, $89,4 \%$ ( $n=311)$ no presentaban enfermedad de base; el $49,7 \%(n=173)$ no utilizaban preservativos en sus relaciones previas al embarazo y sólo el 4,6\% $(n=16)$ manifestó tener más de una pareja sexual en el último año. El 10,6\% ( $n=37)$ presentó una ITS previa que incluyó 26 casos de HPV, 4 hepatitis B, 3 Herpesvirus, 2 C. trachomatis, un caso de hepatitis $C$ y una coinfección HIV-HPV. No se encontró una relación estadísticamente significativa para ninguno de los factores citados anteriormente. La Tabla 1 describe las características demográficas y de comportamiento sexual observadas en la población en estudio.

Tabla 1: Características demográficas y de comportamiento sexual en las embarazadas estudiadas

\begin{tabular}{|c|c|c|c|c|}
\hline Variable & $\begin{array}{c}\begin{array}{c}\text { Total N } \\
(\%)\end{array} \\
\end{array}$ & $\begin{array}{c}\mathbf{C T}+\mathbf{N} \\
(\%)\end{array}$ & $\mathbf{C T}-\mathbf{N}(\%)$ & Valor p \\
\hline \multicolumn{5}{|c|}{ Edad } \\
\hline Hasta 25 años & $30(8,6)$ & $4(13,3)$ & $26(86,7)$ & $<0,001$ \\
\hline$>25$ años & $318(91,4)$ & $3(0,9)$ & $315(99,1)$ & \\
\hline \multicolumn{5}{|c|}{ Nivel educativo } \\
\hline Primario & $11(3,2)$ & $2(18,2)$ & $9(81,8)$ & $<0,001$ \\
\hline 2rio/3rio/Univ & $337(96,8)$ & $5(7,7)$ & $332(92,3)$ & \\
\hline \multicolumn{5}{|c|}{ Edad de inicio de relaciones sexuales } \\
\hline Hasta 18 años & $210(60,3)$ & $7(3,3)$ & $203(96,7)$ & 0,0303 \\
\hline$>18$ años & $138(39,7)$ & $0(0)$ & $138(100)$ & \\
\hline \multicolumn{5}{|c|}{ Nueva pareja sexual en el último año } \\
\hline $\mathbf{S i}$ & $16(4,6)$ & $0(0)$ & $16(100)$ & 0,999 \\
\hline No & $332(95,4)$ & $7(2,1)$ & $325(97,9)$ & \\
\hline \multicolumn{5}{|c|}{ Abortos previos } \\
\hline $\mathbf{S i}$ & $78(22,4)$ & $2(2,6)$ & $76(97,2)$ & 0,49 \\
\hline No & $270(77,6)$ & $5(1,9)$ & $265(98,1)$ & \\
\hline \multicolumn{5}{|c|}{ Síntomas ginecológicos bajos } \\
\hline Si & $88(25,3)$ & $6(6,8)$ & $82(93,2)$ & 0,001 \\
\hline No & $260(74,7)$ & $1(0,4)$ & $259(99,6)$ & \\
\hline \multicolumn{5}{|c|}{ ITS previa } \\
\hline $\mathbf{S i}$ & $37(10,6)$ & $0(0)$ & $37(100)$ & 0,616 \\
\hline No & $311(89,4)$ & $7(2,3)$ & $304(97,7)$ & \\
\hline
\end{tabular}


La Tabla 2 muestra los factores asociados a la prevalencia de $C$. trachomatis en las siete pacientes con un resultado positivo. En cuatro de ellas pudo conocerse el estado y la evolución de los recién nacidos y todas presentaron un embarazo a término; los niños nacidos tuvieron un peso adecuado a la edad gestacional. Los casos positivos recibieron tratamiento de pareja al momento del diagnóstico.

Tabla 2: Factores asociados a la prevalencia de C.trachomatis

\begin{tabular}{|c|c|c|c|c|c|c|c|c|c|c|c|c|c|}
\hline \multirow{2}{*}{ Paciente } & \multirow{2}{*}{ Edad } & \multicolumn{4}{|c|}{ Nivel educativo } & \multirow{2}{*}{ EB } & \multirow{2}{*}{$\begin{array}{c}\text { Inicio } \\
\text { AS }\end{array}$} & \multirow{2}{*}{$\begin{array}{c}\mathbf{N}^{\mathbf{o}} \text { de } \\
\text { parejas } \\
\text { en el último } \\
\text { año }\end{array}$} & \multirow{2}{*}{$\begin{array}{c}\text { ITS } \\
\text { previa }\end{array}$} & \multirow{2}{*}{$\begin{array}{l}\text { Método } \\
\text { barrera }\end{array}$} & \multirow{2}{*}{$\begin{array}{c}\text { Síntomas } \\
\text { ginecológicos }\end{array}$} & \multirow{2}{*}{$\begin{array}{l}\text { Aborto } \\
\text { previo }\end{array}$} & \multirow{2}{*}{$\begin{array}{c}\text { Embarazo } \\
\text { ectópico }\end{array}$} \\
\hline & & 1rio & 2rio & 3rio & Univ. & & & & & & & & \\
\hline 1 & 18 & si & si & no & no & no & 17 & 1 & no & a veces & dispareunia & no & no \\
\hline 2 & 29 & si & si & no & no & $\mathrm{s} / \mathrm{d}$ & 18 & 3 & no & a veces & leucorrea & si & no \\
\hline 3 & 17 & si & si & no & no & no & 15 & 1 & no & a veces & leucorrea/dispareunia & si & no \\
\hline 4 & 29 & si & no & no & no & no & 17 & 1 & no & no & leucorrea & no & no \\
\hline 5 & 31 & si & no & no & no & no & 16 & 1 & no & no & $\mathrm{s} / \mathrm{d}$ & no & no \\
\hline 6 & 20 & si & si & no & no & no & 16 & 1 & no & no & leucorrea & no & no \\
\hline 7 & 21 & si & si & no & no & no & 15 & 1 & no & no & leucorrea & no & no \\
\hline
\end{tabular}

s/d: sin datos; AS: actividad sexual; ITS: infección de transmisión sexual; EB: enfermedad de base

\section{Discusión}

La prevalencia global de la infección por $C$. trachomatis en nuestro grupo de estudio fue del $2 \%$, con un $13,3 \%$ en mujeres menores de 25 años $(p<0,001)$. La revisión de la literatura muestra una clara diferencia de las prevalencias según la media de edad de los grupos estudiados. Cuando las poblaciones presentaron un promedio de edad inferior a los 25 años, las prevalencias observadas fueron significativamente más altas que las de los grupos de mayor edad. Así, nuestro trabajo coincide con los de Pereboom M et al $(1,9 \%){ }^{(20)}$ y Piñero $L$ et al (1\%) ${ }^{(21)}$. En estos reportes, la edad promedio fue superior a los 25 años. Marrama $M^{(22)}$, Borborema-Alfaia A et al (11) y Jalil E et al (23) estudiaron grupos cuyo promedio de edad fue menor a los 25 años y las prevalencias observadas fueron de $10,4 \%, 11 \%$ y $9,4 \%$ respectivamente.

Con respecto al nivel educacional hubo una diferencia estadísticamente significativa entre las pacientes PCR-positivas con estudios primario $18,2 \%$ y secundario o superior $7,7 \%(p<0,001)$. Esta observación coincide con las de otros autores, Borborema-Alfaia A et al (11), Ginige $S$ et al (24), Rours GlJG et al (2), quienes sostienen que la falta de educación y un nivel socioeconómico bajo son factores a considerar al momento de diseñar las políticas de salud pública para prevenir la emergencia de esta enfermedad en toda la población sin diferencias sociales o económicas.

El $85,7 \%$ de nuestras pacientes positivas presentaron algún signo o síntoma genital, que incluyeron leucorrea, dispareunia, prurito o ardor al orinar. Debido a que una alta proporción de pacientes positivas son asintomáticas, la constatación de alguno de estos hallazgos durante el embarazo sugeriría la necesidad de investigar la presencia de esta bacteria para poder implementar el tratamiento adecuado y evitar las consecuencias ulteriores ${ }^{(3,5)}$.

Diversos reportes han demostrado una asociación entre la infección por C. trachomatis durante el primer trimestre y abortos ${ }^{(3)}$. El rol de esta bacteria en etapas más avanzadas del embarazo es menos claro, aunque evidencias serológicas de una infección aguda en este periodo también han sido asociadas con feto muerto y parto pretérmino ${ }^{(25,26)}$. Por estos antecedentes el momento elegido para efectuar el estudio fue el primer trimestre del embarazo.

Si bien la detección precoz de esta bacteria y su rápido tratamiento evitan complicaciones obstétricas y neonatales de importancia, actualmente en Argentina el sistema de salud privado no contempla la cobertura de esta práctica como screening de rutina durante el embarazo. Una limitación del trabajo fue la utilización del primer chorro de orina como muestra. Si bien éste presenta una sensibilidad ligeramente inferior si se compara con el hisopado cervical o vaginal ${ }^{(27)}$, el mismo es un método no invasivo, aceptable para el diagnóstico de infecciones genitales por $C$. trachomatis y es comúnmente usado como técnica de screening durante el embarazo.

El porcentaje de resultados inválidos encontrados en nuestro estudio, 0,57\%, fue menor al hallado en otros realizados en Brasil del 3,7\% ${ }^{(28)}$ y en España del 4,4\% ${ }^{(21)}$. 


\section{Conclusión}

La prevalencia de $13,3 \%$ de $C$. trachomatis en embarazadas menores de 25 años observado en nuestro trabajo, justificaría su búsqueda en este grupo etario debido a que la prevención de esta infección evitará graves complicaciones maternas y neonatales que pueden ser prevenidas con la implementación de un tratamiento oportuno. Asimismo el conocimiento de la prevalencia de esta infección en el ámbito de la salud es esencial para el diseño de un apropiado programa de control de infecciones, especialmente en mujeres jóvenes que corren mayor riesgo de padecerla. Sería fundamental que las autoridades sanitarias definan planes para el diagnóstico precoz de las infecciones causadas por esta bacteria de comportamiento silencioso pero agresivo.

\section{Bibliografía}

1. Estrategia mundial del sector de la salud contra las infecciones de transmisión sexual 2016-2021 hacia el fin de las ITS. Junio 2016 Disponible en: http://apps.who.int/iris/bitstream/handle/10665/250253/WHO-RHR-16.09spa.pdf

2. Rours GIJG, Duijts L, Moll HA, et al. Chlamydia trachomatis infection during pregnancy associated with preterm delivery: a population-based prospective cohort study. European Journal of Epidemiology. 2011;26(6):493-502. doi:10.1007/s10654-011-9586-1.

3. Schmidt R, Muniz RR, Cola E, Stauffert D, Silveira MF, Miranda AE. Maternal Chlamydia trachomatis Infections and Preterm Births in a University Hospital in Vitoria, Brazil. Zakar T, ed. PLoS ONE. 2015;10(10):e0141367. doi:10.1371/journal.pone.0141367.

4. Jolande A. Land, Anna P. Gijsen, Johannes LH Evers, Cathrien A. Bruggeman; Chlamydia trachomatis en mujeres subfértiles sometidas a instrumentación uterina: Screen or treat?, Human Reproduction, Volumen 17, Número 3 , 1 de marzo de 2002, páginas 525-527. https://doi.org/10.1093/humrep/17.3.525

5. Eggert-Kruse W, Rohr G, Demirakca T, Rusu R, Naher H, Petzoldt D, Runnebaum B. 1997.Chlamydial serology in 1303 asymptomatic subfertile couples. Hum Reprod 12:1464-1475. doi:10.1093/humrep/12.7.1464.

6. Jackson Y, Sebo P, Aeby G, Bovier P, Ninet B, Schrenzel J, Sudre $P$, et al. Prevalence and associated factors for Chlamydia trachomatis infection among undocumented immigrants in a primary care facility in Geneva, Switzerland: a cross-sectional study. J Immigr Minor Health. 2010 Dec;12(6):909-14. doi: 10.1007/s10903-0109354-4.

7. Ruiz, A. et al. Estudio piloto de prevalencia de infección por Chlamydia trachomatis detectada por PCR en mujeres con parto prematuro en el Instituto Materno Infantil de Bogotá. Revista Colombiana de Obstetricia y Ginecología, [S.I.], $\quad$ v. 56, n. 3 , p. 225-230, sep. $2005 . \quad$ ISSN 2463-0225. Disponible en: $<$ https://revista.fecolsog.org/index.php/rcog/article/view/529>.

8. Peuchant $O$, et al .Screening for Chlamydia trachomatis, Neisseria gonorrhoeae, and Mycoplasma genitalium should it be integrated into routine pregnancy care in French young pregnant women? Diagn Microbiol Infect Dis. 2015 May;82(1):14-9. doi: 10.1016/j.diagmicrobio.2015.01.014. Epub 2015 Feb 3.

9. Mårdh PA. Influence of infection with Chlamydia trachomatis on pregnancy outcome infant health and life long sequelae in infected offspring. Best Pract Res Clin Obstet Gynaecol. 2002 Dec;16(6):847-64. https://doi.org/10.1053/beog.2002.0329

10.CDC. Sexually Transmitted Diseases Treatment Guidelines, 2015. June 5, 2015 / 64(RR3);1-137

11.Borborema-Alfaia Ana Paula B. de, Freitas Norma Suely de Lima, Astolfi Filho Spartaco, Borborema-Santos Cristina Maria. Chlamydia trachomatis infection in a sample of northern Brazilian pregnant women: prevalence and prenatal importance. Braz J Infect Dis [Internet]. 2013 Oct [cited 2018 May 20] ; 17( 5 ): 545-550. Available from: $\quad h$ ttp://www.scielo.br/scielo.php?script=sci_arttext\&pid=S1413-86702013000500006\&/ng=en. http://dx.doi.org/10.1016/j.bjid.2013.01.014.

12. Cacho J, Sanz F, Blanco MA. La enfermedad silenciosa por Chlamydia trachomatis: necesidad urgente de detección y tratamiento en mujeres. Enferm Infecc Microbiol Clin 2001;19:419-21 - DOI: 10.1016/S0213005X(01)72684-5

13. Kimberly A. Workowski, Stuart M. Berman; Centers for Disease Control and Prevention Sexually Transmitted Disease Treatment Guidelines, Clinical Infectious Diseases, Volume 53, Issue suppl_3, 15 December 2011, Pages S59-S63, https://doi.org/10.1093/cid/cir694

14. Farinati $A$, et al. Infecciones asintomáticas por Chlamydia trachomatis: un problema controlable en la población adolescente. Rev Panam Infectol 2008;10(1):8-12

15. Occhionero Marcelo, Paniccia Laura, Pedersen Dina, Rossi Gabriela, Mazzucchini Héctor, Entrocassi Andrea et al. Prevalencia de la infección por Chlamydia trachomatis y factores de riesgo de infecciones transmisibles sexualmente en estudiantes universitarios. Rev. argent. microbiol. [Internet]. 2015 Mar [citado 2018 Mayo 20]; 47( 1 ): 9-16. Disponible en: http://www.scielo.org.ar/scielo.php?script=sci_arttext\&pid=S032575412015000100003\&Ing=es. http://dx.doi.org/10.1016/j.ram.2014.11.003.

16. Ovalle Alfredo, Martínez María A, de la Fuente Felipe, Falcon Nicolás, Feliú Felipe, Fuentealba Felipe et al. Prevalencia de infecciones de transmisión sexual en mujeres embarazadas atendidas en un hospital público de Chile. Rev. chil. infectol. [Internet]. 2012 Oct [citado 2018 Mayo 20] ; 29( 5 ): 517-520. Disponible en: 
https://scielo.conicyt.cl/scielo.php?script=sci_arttext\&pid=S0716-10182012000600006\&lng=es.

http://dx.doi.org/10.4067/S0716-10182012000600006.

17. Cabeza J, García P, Segura E, et al. Feasibility of Chlamydia trachomatisscreening and treatment in low-risk pregnant women in Lima, Peru: a prospective study in two large urban hospitals. Sexually transmitted infections. 2015;91(1):7-10. doi:10.1136/sextrans-2014-051531.

18. Silveira Mariângela Freitas da, Sclowitz lândora Krolow Timm, Entiauspe Ludmila Gonçalves, Mesenburg Marilia Arndt, Stauffert Dulce, Bicca Guilherme Lucas de Oliveira et al. Chlamydia trachomatis infection in young pregnant women in Southern Brazil: a cross-sectional study. Cad. Saúde Pública [Internet]. 2017 [cited 2018 May 20]; $33(1$ ): e00067415. Available from: http://www.scielo.br/scielo.php?script=sci_arttext\&pid=S0102311X2017000105011\&lng=en. Epub Feb 13, 2017. http://dx.doi.org/10.1590/0102-311x00067415.

19. Di Bartolomeo S, Rodríguez M, Sauka D, Alberto De Torres R. Enferm Infecc Microbiol Clin. 2001 Mar;19(3):99102. https://doi.org/10.1016/S0213-005X(01)72577-3

20.Pereboom, MT, et al.Knowledge and acceptability of Chlamydia trachomatis screening among pregnant women and their partners; a cross-sectional study. BMC Public Health 2014 14:704. http://doi.org/10.1186/1471-2458-14704

21. Piñeiro L, Lekuona A, Cilla G, et al. Prevalence of Chlamydia trachomatisinfection in parturient women in Gipuzkoa, Northern Spain. SpringerPlus. 2016;5:566. doi:10.1186/s40064-016-2268-4.

22. Marramá M. (2016) "Prevalencia de Chlamydia trachomatis en mujeres que cursan el tercer trimestre de embarazo asistidas en centros de salud municipales de la ciudad de Córdoba". Tesis de Maestría en Salud Pública. Facultad de Ciencias Médicas. UNC. Córdoba, Argentina

23. Jalil Emilia Moreira, Pinto Valdir Monteiro, Benzaken Adele Schwartz, Ribeiro Denis, Oliveira Eduardo Campos de, Garcia Enrique Galban et al. Prevalência da infecção por clamídia e gonococo em gestantes de seis cidades brasileiras. Rev. Bras. Ginecol. Obstet. [Internet]. 2008 Dez [citado 2018 Maio 20] ; 30(12): 614-619. Disponível em: $\quad h t t p: / / w w w . s c i e l o . b r / s c i e l o . p h p ? s c r i p t=s c i \_a r t t e x t \& p i d=S 0100-72032008001200005 \& l n g=p t$. http://dx.doi.org/10.1590/S0100-72032008001200005.

24. Ginige S, Fairley CK, Hocking JS, Bowden FJ, Chen MY. Interventions for increasing chlamydia screening in primary care: a review. BMC Public Health. 2007;7:95. doi:10.1186/1471-2458-7-95.

25. Kortekangas-Savolainen O. Mäkinen J. Koivusalo K. Mattila K. Hospital-Diagnosed Late Sequelae after Female Chlamydia trachomatis Infections in 1990-2006 in Turku, Finland. Gynecol Obstet Invest 2012;73:299-303 https://doi.org/10.1159/000334822

26. Paredes Q V. Chlamydia Trachomatis y Embarazo Ectópico. Revista HCUCh 2006; 17: 5 - 12

27.CDC. Recommendations for the Laboratory-Based Detection of Chlamydia trachomatis and Neisseria gonorrhoeae - 2014. March 14, 2014 / 63(RR02);1-19

28. Pinto Valdir Monteiro, Tancredi Mariza Vono, Silva Roberto Jose de Carvalho da, Khoury Zarifa, Buchalla Cássia Maria. Prevalence and factors associated with Chlamydia trachomatis infection among women with HIV in São Paulo. Rev. Soc. Bras. Med. Trop. [Internet]. 2016 June [cited 2018 May 20]; 49( 3 ): 312-318. Available from: http://www.scielo.br/scielo.php?script=sci_arttext\&pid=S0037-86822016000300312\&lng=en.

http://dx.doi.org/10.1590/0037-8682-0169-2016. 